\title{
Improper hydration induces global gene expression changes associated with renal development in infant mice
}

\author{
Chong-Su Kim ${ }^{1}$ and Dong-Mi Shin ${ }^{1,2^{*}}$
}

\begin{abstract}
Background: The kidney is a major organ in which fluid balance and waste excretion is regulated. For the kidney to achieve maturity with functions, normal renal developmental processes need to occur. Comprehensive genetic programs underlying renal development during the prenatal period have been widely studied. However, postnatal renal development, from infancy to the juvenile period, has not been studied yet. Here, we investigated whether structural and functional kidney development was still ongoing in early life by analyzing the renal transcriptional networks of infant (4 weeks old) and juvenile (7 weeks old) mice. We further examined the effects of dehydration on kidney development to unravel the mechanistic bases underlying deteriorative impact of pediatric dehydration on renal development.
\end{abstract}

Methods: 3-week-old infant mice that just finished weaning period were provided limited access to a water for fifteen minutes per day for one week (RES 1W) and four weeks (RES 4W) to induce dehydration while control group consumed water ad libitum with free access to the water bottle. Transcriptome analysis was conducted to understand physiological changes during postnatal renal development and dehydration.

Results: Kidneys in 4-week- and 7-week-old mice showed significantly distinctive functional gene networks. Gene sets related to cell cycle regulators, fetal kidney patterning molecules, and immature basement membrane integrity were upregulated in infantile kidneys while heightened expressions of genes associated with ion transport and drug metabolism were observed in juvenile kidneys. Dehydration during infancy suppressed renal growth by interrupting the SHH signaling pathway, which targets cell cycle regulators. Importantly, it is likely that disruption of the developmental program ultimately led to a decline in gene expression associated with basement membrane integrity.

Conclusions: Altogether, we demonstrate transcriptional events during renal development in infancy and show that the impacts of inadequate water intake in the early postnatal state heavily rely on the impairment of normal renal development. Here, we provide a meaningful perspective of renal development in infancy with a molecular and physiological explanation of why infants are more vulnerable to dehydration than adults. These results provide new insights into the molecular effects of dehydration on renal physiology and indicate that optimal nutritional interventions are necessary for pediatric renal development.

Keywords: Dehydration, Infancy, Kidney transcriptome, Renal development,

\footnotetext{
* Correspondence: shindm@snu.ac.kr

'Department of Food and Nutrition, Seoul National University, Seoul 08826,

South Korea

${ }^{2}$ Research Institute of Human Ecology, Seoul National University, Seoul

08826, South Korea
} 


\section{Background}

The kidney is a major organ that plays crucial roles in homeostasis in the body. It filters the blood to excrete waste products from the body [17] and controls body fluid volume, solute, and acid balance within a tight range in cooperation with other endocrine organs [33]. The critical functions of the kidney in the fluid regulatory system are implicated in precise regulation of plasma osmolality. When the body senses changes in osmolality, osmoreceptors at the hypothalamus stimulate arginine vasopressin (AVP) release, which promotes fluid reabsorption in the kidney and thirst by which water retention increases [30].

Kidney organogenesis comprises several common developmental steps: proliferation and differentiation of stem cells, epithelial-mesenchymal cell transition, branching, and regional segmentation [13, 16, 46]. Complex gene regulatory networks mediate cell interactions underlying anatomical and functional modifications in kidney morphogenesis [23, 45]. Especially, dynamic regulations underlying cell and tissue integrity are essential in kidney function and are highly associated with renal disorders [49]. A highly selective permeable glomerular barrier membrane establishes networks with podocyte foot processes and slit diaphragm, a modified form of adherens junction, to gain mature filtration functions [36]. Thus, retaining a stable cell and junction architecture is required for normal renal function [26, 36, 49].

Body water constitutes cells and tissues and functions as a carrier of nutrients and excretions [17, 29]. It also serves as both reactant and product in metabolisms [17]. Optimal hydration is necessary for a healthy life [9]. It is well documented that dehydration negatively impacts multiple physiological disorders $[9,17,33]$. Healthy adults regulate fluid balance to maintain homeostasis whereas infants have an immature fluid regulatory system [37] with a higher body water composition, reaching $75 \%$ [9]. Moreover, infants, right after weaning, are more prone to drink insufficient water since caregivers are likely to be unaware of their thirst [9]. In this respect, infants seem to be at higher risk for dehydration.

While recent studies have shown that further growth of the nephrons and elongation of tubular structures occur after birth [8], little is known about how genetic events are regulated in renal growth postnatally $[2,7$, 24, 50]. Moreover, the molecular and physiological background for why infants are more vulnerable to dehydration has not been studied yet, and there are few studies on whether dehydration causes physiological changes in infants. We hypothesized that fundamental mechanisms of pediatric dehydration may underlie renal developmental processes. To confirm this assumption, we studied the effects of insufficient water intake in infant mice, with emphasis on physiological and transcriptional changes in the developing kidney.
In this study, we performed global gene expression profiling analysis and identified the functional networks of differentially expressed genes in the kidneys of infant and juvenile mice. We showed that kidneys undergo biological processes to achieve structural and functional maturation even after birth and dehydration altered gene networks associated with normal renal development and maturation. These results may provide evidence that sufficient water intake from infancy to juvenile period is necessary for normal renal physiology and system development.

\section{Methods \\ Experimental animals}

Right after weaning, 3-week-old male C57BL/6 mice were housed for 1 and 4 weeks and sacrificed at the age of 4 and 7 weeks old, corresponding to infant and juvenile. Animals were randomly assigned into control $(\mathrm{CON})$ and water restriction group (RES). A total of four groups were used in comparison analysis: CON $1 \mathrm{~W}$ (4 weeks old, $n=5$ ), CON $4 \mathrm{~W}$ (7 weeks old, $n=6$ ), RES $1 \mathrm{~W}$ (4 weeks old, $n=6$ ), and RES 4W (7 weeks old, $n=8$ ). Animals were maintained in a 12-h light/dark cycle and fed ad libitum with an AIN93G diet. Animals from water restriction group were maintained dehydrated until they were sacrificed, $24 \mathrm{~h}$ after the last provision of water bottle. All experimental procedures were approved by the IACUC (Institutional Animal Care and Use Committee) of Seoul National University and conducted according to the IACUC guidelines.

\section{Water restriction}

Animals in water restriction group were imposed limited access to water. A water bottle was given to animals for 15 min a day during the experimental period. Control mice consumed water ad libitum with free access to the water bottle.

\section{Plasma biochemical analysis}

Serum biochemical analysis for blood urea nitrogen (BUN) and serum creatinine ( $\mathrm{sCr}$ ) was conducted using a drychemistry blood analyzer, Spotchem SP-4410 (Arklay, Kyoto, Japan). Plasma osmolality was determined by Fiske 210 Micro-Sample Osmometer (Fiske, Norwood, MA, USA).

\section{RNA isolation}

Total RNA was extracted from the kidneys and brain using DNA-free RNA isolation kit (RNAqueous-4PCR kit; Ambion, Austin, TX) according to the manufacturer's directions. Total RNA integrity and quantity were assessed with a NanoDrop 2000 Spectrophotometer (Thermo Fisher Scientific, Wilmington, DE). 


\section{Microarray hybridization}

RNA samples were amplified for microarray analyses using Illumina TotalPrep RNA Amplification Kit (Ambion, Austin, TX). Five hundred nanograms of total RNA was used to prepare labelled complementary RNA (cRNA) with overnight incubation. Amplified cRNA was hybridized on Illumina MouseWG-6 Expression BeadChip arrays. The arrays were scanned with BeadArray Reader (BeadStation 500 G Instrument, Illumina Inc.). Identification and quantification of spot images were obtained by Genome Studio software v1.0.2 (Illumina Inc.).

\section{Bioinformatic analysis of microarray data}

The analysis was performed as described in [41]. Raw data was log-transformed and normalized by quantile normalization using Genome Studio software (Illumina Inc.). Differentially expressed genes among four groups were identified using an ANOVA $(p<0.05)$ by Partek $^{\circ}$ Genomics Suite software v6.6 (Partek, St Louis, MI) (http:// www.partek.com/partekgs). Average expression levels of genes were compared between groups, and calculated $p$ values were corrected for multiple comparisons using false discovery rate algorithm. Significant genes with fold change $>1.5$ and FDR $<0.01$ were used for further analysis.

Hierarchical clustering analysis was performed using the Pearson correlation distance matrix with average linkage algorithm in Genesis software v1.7.5 [43]. Functional categories of significant genes were determined by a right-tailed Fisher exact test. To examine the significance of functional categories which were classified based on Ingenuity Knowledge Base, Gene Set Enrichment Analysis was carried out (http://www.broadinstitute.org/ gsea/index.jsp). Mechanistic networks underlying signaling pathways and metabolic pathways were built based on the Ingenuity Knowledge Base. Upstream regulator analysis was carried out to predict upstream regulators in direct or indirect relationships with dataset using Ingenuity Pathway Analysis (IPA) [22]. Functional network map of gene sets was constructed by using CytoScape software v3.2.0 (http:// cytoscape.org) [40]. The microarray dataset is available at the Gene Expression Omnibus (www.ncbi.nlm.nih.gov/geo/ , accession number GSE75604).

\section{Quantitative RT-PCR}

DNase I treated total RNA was converted into cDNA by two-step procedure with MessageSensor RT kit (Ambion, Austin, TX), and messenger RNA (mRNA) levels were quantified by SYBR-GREEN qPCR method (Applied Biosystem, Carlsbad, CA). Relative mRNA expression level was calculated by $\Delta \Delta C_{T}$ method. Glyceraldehyde-3-phosphate dehydrogenase (GAPDH) was used as a housekeeping gene for normalization of mRNA expression of each sample. Primer sequences are as follows: Avp: forward 5'-CCAGGATGCTCAAC
ACTACG-3', reverse 5' -CTCTTGGGCAGTTCTGGA AG-3'; Ccnd1: forward 5' -CGTGGCCTCTAAGATGA AGG-3', reverse 5' -CTGGCATTTTGGAGAGGAAG3'; Cdkn2b: forward 5'-AGATCCCAACGCCCTGAA C-3', reverse 5' -CGCAGTTGGGTTCTGCTC-3'; and Gapdh: forward 5'-TGCACCACCAACTGCTTAG-3', reverse 5'-GATGCAGGGATGATGTTC-3'.

\section{Western blot analysis}

Proteins were extracted from the kidneys. Equal amounts of protein were subjected to SDS-PAGE and transferred to PVDF membranes. The membranes were incubated with anti-SMO (1:10000, ab72130) and antiGLIS1 (1:1000, ab105873) primary antibodies from Abcam (Abcam Inc., Cambridge, MA). Anti- $\alpha$-tubulin (1:10000, T5168) from Sigma (Sigma Chemical Co., St. Louis, MO) was used as control. Horseradish peroxidaseconjugated goat anti-rabbit secondary antibody (1:5000, \#7074S) from Cell Signaling Technology (Cell Signaling Technology Inc., Danvers, MA) and goat anti-mouse secondary antibody (1:10000, G21040) from Invitrogen (Invitrogen, Carlsbad, CA) were used for detection. Protein bands were visualized using ECL Western blot detection reagents (Amersham Pharmacia Biotech, Piscataway, NJ).

\section{Statistical analysis}

Data are expressed as mean \pm SEM. Statistical significance $(p<0.05)$ was evaluated by unpaired Student's $t$ test between two groups. Statistical analyses were performed using GraphPad Prism 6 software (GraphPad Software Inc., La Jolla, CA).

\section{Results}

Time-limited access to water bottle induces mild dehydrating physiological conditions in infant mice Infant mice that just finished weaning were provided limited access to a water bottle for 15 min per day for 1 week (RES 1W) and 4 weeks (RES 4W). Mice in the RES 1W group consumed 2.4 times less water compared to the age-matched control mice in the CON $1 \mathrm{~W}$ group with ad libitum water intake $(p<0.001)$ (Fig. 1a). Mice in the RES $4 \mathrm{~W}$ that grew into juveniles with the same daily waterrestriction treatment showed a significant reduction in daily water intake, on average down to a third of that in control mice $(\mathrm{CON} 4 \mathrm{~W})(p<0.001)$. In order to verify whether the reduction in water intake was sufficient to induce physiological changes in those mice, we determined the levels of plasma osmolality. Mice in RES $1 \mathrm{~W}$ had significantly higher plasma osmolality than mice in CON $1 \mathrm{~W}$ $(p<0.001)$. The 4-week dehydration resulted in increased plasma osmolality as well, although it was not significant $(p=0.083)$ (Fig. 1b). Next, we measured the transcript level of vasopressin in the brain and found dramatic 


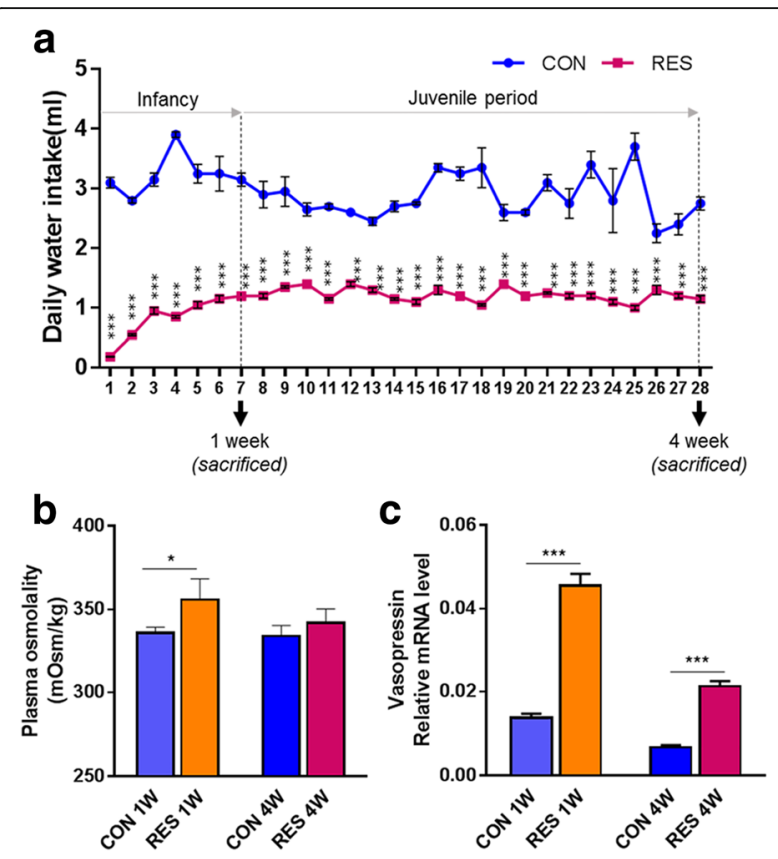

Fig. 1 Mild dehydration was induced in infant mice. To observe the effects of prolonged dehydration on development, an experiment was performed from infancy to the juvenile period. The restriction group had limited access to the water bottle for 15 min a day. The water restriction group had reduced daily water intake compared to the control group (a). Mild dehydration was generated, resulting in elevated plasma osmolality (b) and brain vasopressin mRNA level (c) in the dehydrated mice. The number of samples in each group is as follows: CON 1W $(n=4)$, RES $1 \mathrm{~W}(n=4)$, CON 4W $(n=3)$, and RES 4W $(n=4)$. Data are expressed as mean \pm SEM. Student's $t$ test; ${ }^{* *} p<0.01,{ }^{* *} p<0.001$ versus control group increases in vasopressin production in both RES $1 \mathrm{~W}$ and RES $4 \mathrm{~W}$, compared to CON $1 \mathrm{~W}$ and CON $4 \mathrm{~W}$, respectively $(p<0.001)$ (Fig. 1c).

\section{Transcriptome profiling analysis of the kidneys in dehydrated mice}

To examine the effect of dehydration in the developing mouse, the organ weight was measured. The weight of the kidneys increased with age from $0.22 \mathrm{~g}$ in CON $1 \mathrm{~W}$ to $0.28 \mathrm{~g}$ in $\mathrm{CON} 4 \mathrm{~W}(p<0.001)$; however, dehydration caused a decrease of kidney weight in infant mice (RES $1 \mathrm{~W})$ by $22 \%(p<0.01)$ and juvenile mice (RES $4 \mathrm{~W})$ by $8 \%(p=0.23)$ compared to age-matched controls (Fig. 2a). To understand the molecular events underlying kidney growth and development from the age of infancy to the juvenile stage during the life cycle and to unveil the mechanism by which the kidneys' weight were reduced while developing mice were under dehydration status, we conducted gene expression profiling analysis of kidneys using a genome-scale microarray.

Principal component analysis (PCA) showed that the transcriptome profiles were readily distinguished by either

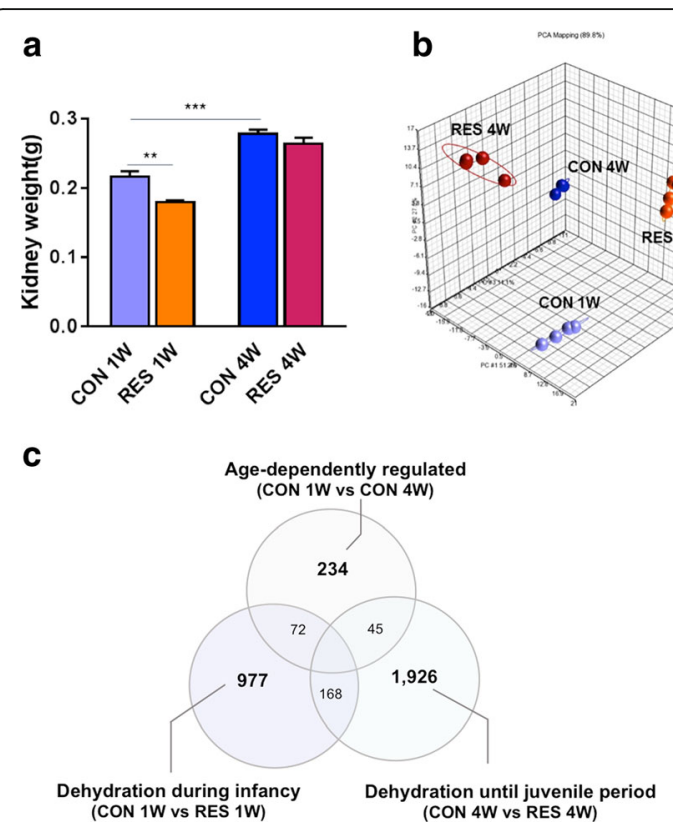

Fig. 2 Transcriptome profiling analysis of the kidneys in dehydrated and control mice using genome-scale Illumina microarray. a The weight of the kidney increased with age, but dehydration caused decreased weight in the kidneys of infant mice (RES 1W) by $22 \%$ and in juvenile mice (RES $4 \mathrm{~W}$ ) by $8 \%$. b A 3D principal component analysis (PCA) plot of the renal transcriptome data represents significantly distinguishable profiles for each group: CON 1W (light blue), RES 1W (orange), CON 4W (blue), and RES 4W (red). Each dot indicates an individual kidney sample. c The Venn diagram shows the number of probes that were detected with different signal intensity in each dataset. Data are expressed as mean \pm SEM. Student's $t$ test; ${ }^{* *} p<0.01,{ }^{* *} p<0.001$ versus control group age (CON 1W and CON 4W) or a period of sustained water restriction (RES 1W and RES 4W) (Fig. 2b). Differentially expressed genes among the four groups were identified by ANOVA analysis $(p<0.05)$. A total of 234 probes were differentially expressed depending on age in the normal control groups (CON 4W vs. CON 1W). Furthermore, 977 probes were detected to distinctively mediate the transcriptional events in the infantile kidneys of the dehydration group (RES 1W vs. CON 1W), while 1926 differentially expressed probes were in the juvenile kidneys of the dehydration group (RES 4W vs. CON 4W) (Fig. 2c).

\section{Normal infant and juvenile mice present distinct renal transcriptional profiles}

Hierarchical clustering analysis showed that postnatal kidneys at different time points (CON $1 \mathrm{~W}$ and CON 4W) had distinguishable transcriptome profiles (Fig. 3a). Two hundred thirty-four probes were differentially expressed in a comparison of CON $1 \mathrm{~W}$ and CON 4W, with 149 probes upregulated in the kidneys of infant mice $(\mathrm{CON} 1 \mathrm{~W})$ and 85 probes upregulated in juvenile mice $(\mathrm{CON} 4 \mathrm{~W})$ : gene list is given in Additional file 1. 
a

\section{Infantile Kidney}

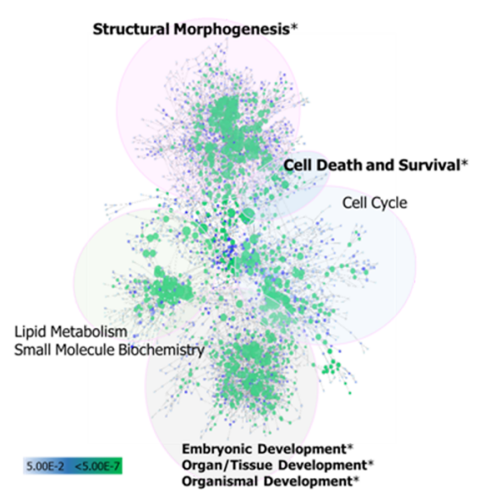

Juvenile Kidney

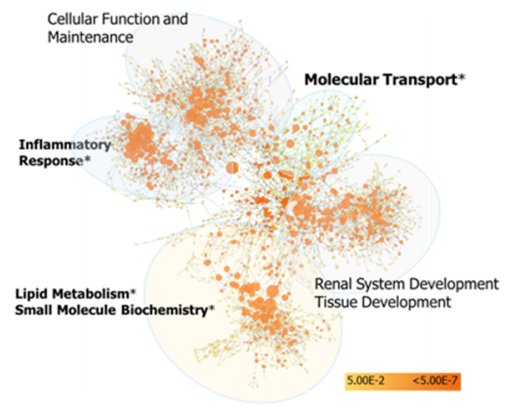

b

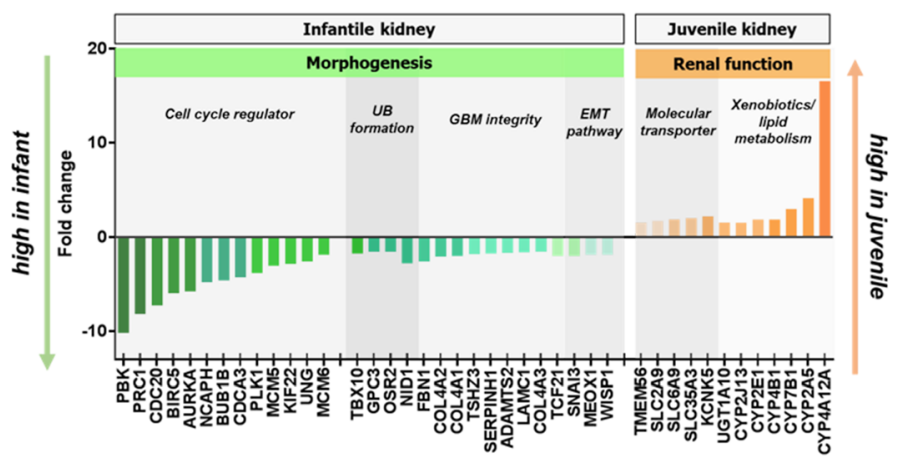

C

Aquaporins

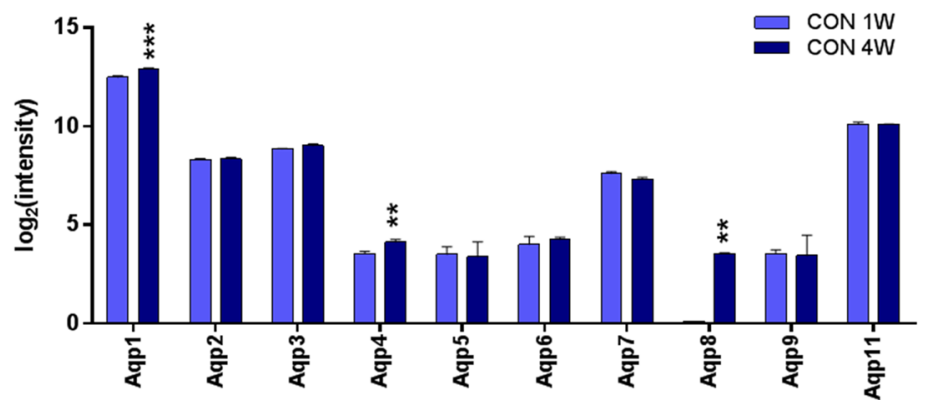

Fig. 3 Distinctive functional network of genes in normal renal development during postnatal life. a Functional network of gene sets of CON 1W (4 weeks old; infant) and CON 4W (7 weeks old; juvenile). Gene ontology (GO) was categorized based on Ingenuity Pathway Analysis (IPA) knowledge. GO terms in bold with asterisk indicate enriched biological functions in gene set enrichment analysis. Each node represents a biological function and its color and size indicate the $p$ value and number of genes in the cluster, respectively. $\mathbf{b}$ Identification of significant genes related to postnatal renal development. $\mathbf{c}$ The increasing transcript levels of aquaporin family with age support that functional maturation were taking place in the kidneys of infant and juvenile mice. UB ureteric bud, GBM glomerular basement membrane, EMT epithelial-mesenchymal transition. Data are expressed as mean \pm SEM. Student's $t$ test; ${ }^{*} p<0.05,{ }^{* *} p<0.01,{ }^{* * *} p<0.001$

This finding implies that the kidneys of infant and juvenile mice might undergo different biological processes.

To extend our knowledge about the biological process occurring in the infantile kidney, we established a functional network map of differentially expressed genes and assigned gene ontology (GO) terms. Enriched GO categories from gene set enrichment analysis revealed that genes related to the cell cycle, tissue morphology, and development were significantly upregulated, whereas they were downregulated in CON 4W (Fig. 3a). Genes associated with cell cycle, which was the most significant biological process in the infantile kidneys, including Aurka, Birc5, Bub1b, Cdc20, Meox1, Pbk, Kif22, Mcm5, Mcm6, Ncaph, Plk1, Ung, and Cdca3 were overexpressed (Fig. 3b), indicating that developmental cellular processes were still abundant in the kidneys of CON $1 \mathrm{~W}$. Among those genes, Aurka, Plk1, Mcm5, and Kif22 have been found to function in embryonic renal development by mediating cell division [21]. In addition, Birc5, which had about a sixfold increase in infant mice, has been reported to be expressed 
only in the tubules and glomeruli of fetal kidney [39]. Meox, with a twofold increase in CON $1 \mathrm{~W}$ compared to CON 4W, is one of the important factors in kidney formation and has a role in epithelial-mesenchymal cell interactions [34]. Genes that are essential for DNA synthesis and cell division (Nasp, Rrm1, Rrm2, Prc1) were upregulated and then eventually downregulated with age in our dataset. Many of the predictive upstream regulators within gene sets of CON $1 \mathrm{~W}$ were associated with cell cycle regulation, including Myc, Tp53, Foxm1, Ccnd1, and Smad3 (Additional file 2). $M y c$ is highly expressed in the metanephros during renal organogenesis, and its disruption is associated with reduced cell proliferation [1]. These results imply that pathways mediating cell cycle progression were dominantly engaged in the development of infantile kidneys.

We also observed that the cluster of genes participating in basement membrane integrity was highly expressed in infant kidneys compared to juvenile kidneys. Genes encoding extracellular matrix (ECM) components are well known to change their expression levels during kidney tubulogenesis and maturation [31]. Among them, collagen is a major component that provides structural integrity and its subtypes appear to have different expression levels during glomerular structural development in the kidney [31]. In our dataset, the members of the collagen IV family (Col4a1, Col4a2) showed two times higher expression in infant than juvenile kidneys (Fig. 3b). In concert with the overexpression of basement membrane composing protein, there was elevated expression of Adamts2, Nid1, Serpinh1, and Wisp1 which participate in ECM synthesis [3, 25] (Fig. 3b). Genetic events regulating ECM deposition demonstrate that improvement of basement membrane integrity occurs in infants, which accounts for structural maturation after birth. In addition, genes that play roles in ureteric bud formation such as Tbx10, Gpc3, Osr2, and Nid1 had higher expression in CON $1 \mathrm{~W}$ than in CON 4W group. Snail is known to regulate mesenchymal to epithelial transformation [5]. Diminishing expression of Snail along with renal maturation in our dataset was coincident with previous work showing that Snail becomes inactive in the mature organ. Taken together, the results indicate that structural development of the kidney is not completed before birth, rather postnatal kidneys continue to grow during infancy and they undergo the processes necessary for mature structural integrity.

Contrary to the observation that structural morphogenesis was the most significant biological process in the transcriptome analysis of kidneys in CON 1W, upregulated genes in the CON 4W kidneys were associated with molecular transport, renal system development and function, and cellular function and maintenance (Fig. 3a). This indicates that functional maturation of the kidney was ongoing followed by morphological development. Genes involved in molecular transport associated with the renal system (Kcnk5, Slc2a9, Slc6a9, Slc35a3) were highly expressed with 1.6- to two-fold changes (Fig. 3b). The cytochrome P450 family, involved in bile acid metabolism in extrahepatic tissues, xenobiotics metabolism (Cyp4b1, Ugt1a10), and steroids and fatty acid metabolism (Cyp2e1, Cyp4b1, Cyp7b1, Cyp4a12a), was also upregulated during the juvenile period (Fig. 3b). These results indicate that the kidneys of juveniles are in the process of developing into functionally matured adult kidneys. We also checked whether aquaporin system that is critical for water transport in the kidney is in development and maturation. The increasing transcript levels of aquaporin family (Aqp1, Aqp4, and Aqp8; $p<0.01$ ) with age support that functional maturation were taking place in the kidneys of infant and juvenile mice although the difference was slight (Fig. 3c).

\section{Dehydrated infant mice show altered Shh signaling pathway associated with impaired renal growth and development}

Dehydration resulted in dramatic changes in kidney transcriptome profiles in infant mice. Each functional category of differentially expressed genes in the RES $1 \mathrm{~W}$ group compared to CON $1 \mathrm{~W}$ was tested for statistical significance. We found that the cell cycle, organ development, and renal system development were in the top seven significant categories (Fig. 4a). Gene set enrichment analysis (GSEA) confirmed that the cell cycle was the most enriched biological process regulated by hydration (Fig. 4b). These findings suggest that dehydration might have caused a deterioration in renal growth.

We further performed pathway analysis and found that one of the most crucial molecular pathways that were significantly regulated by dehydration during kidney organogenesis was the Sonic hedgehog (Shh) pathway. It is known to have a role in the expression of GLI transcription factors whose targets are cell cycle regulators [45]. Interestingly, a significant reduction in the Shh signaling pathway was observed following insufficient water intake. In concert with this, decreased expression of downstream target genes including cell cycle regulators (Cdk1, Cdc20, Ccnd1, Plk1, Bub1b, Rrm2, Mcm5, Mcm6) and cell differentiation-related molecules (Id1, Id2, Rad9, Birc5) was detected in the RES $1 \mathrm{~W}$ group (Fig. 4c). Immunoblotting assays were conducted to validate the microarray analysis results for Shh proteins. SHH signaling molecules such as SMO and GLIS1 had a tendency to be reduced in the RES 1W group compared to the control group $(p=0.35)$ at the protein level (Fig. 4d). Target molecules in the SHH signaling, $C c n d 1$ (1.7-fold decrease) and $C d k n 2 b$ (2.8-fold increase), were shown to be differentially regulated in the RES group by qPCR analyses $(p<0.05)$ (Fig. $4 \mathrm{e})$. 


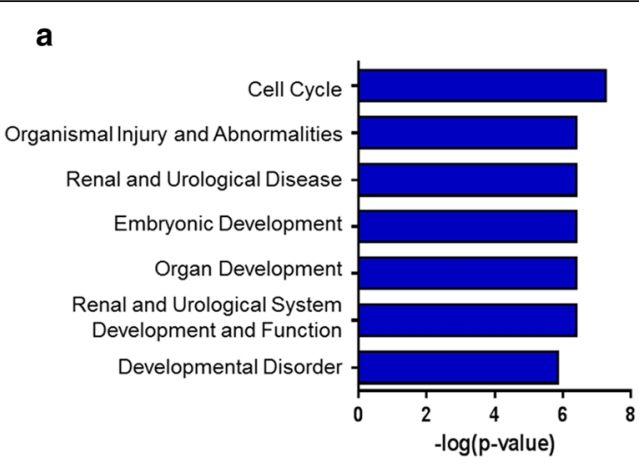

b

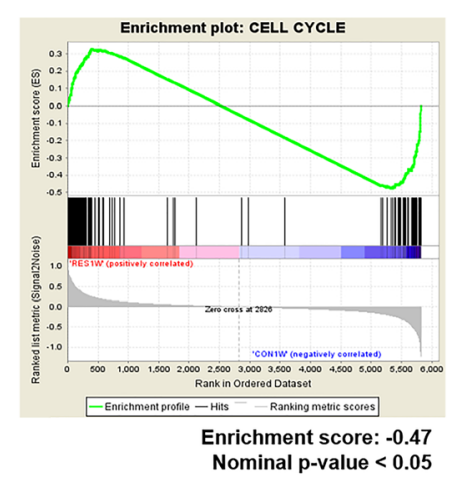

C

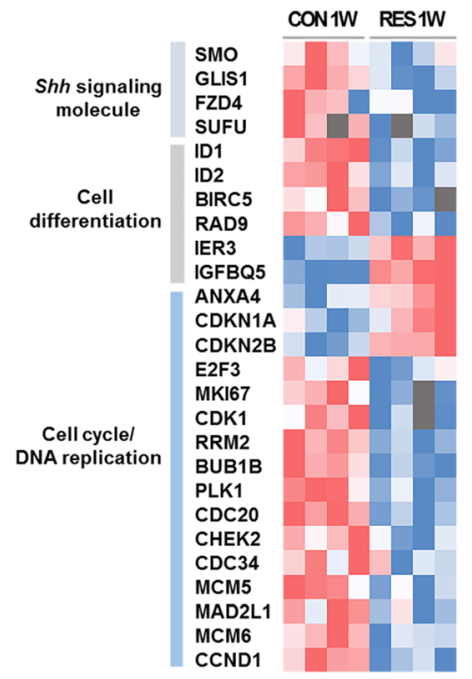

e
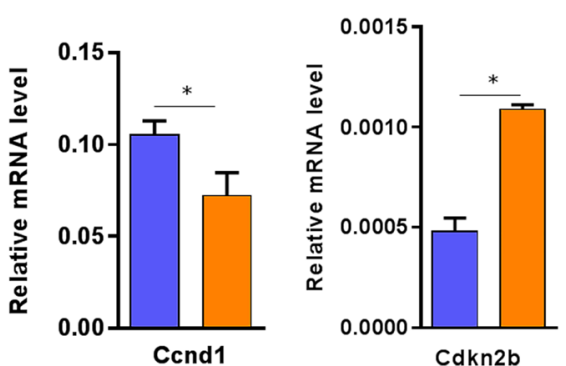

Fig. 4 Dehydration interrupted renal morphogenesis by regulating Shh signaling in infant mice. a Top disease and functional categories of gene sets among differentially expressed genes in dehydrated mice during infancy. b Gene set enrichment analysis (GSEA) was used to evaluate the most significant functional category of differentially expressed genes by dehydration in infant mice and generate enrichment plot of cell cyclerelated genes. c The heatmap shows downstream target genes of the SHH signaling pathway and indicates that cell cycle and cell differentiation regulators were distinctively regulated by dehydration. $\mathbf{d}$ Western blot assay was conducted for SHH signaling members. e The transcript level of its target molecules was validated with qPCR analysis. Data are expressed as mean \pm SEM. Student's $t$ test; ${ }^{*} p<0.05$ versus control group

Dysregulation of transcriptional network associated with basement membrane integrity in juvenile mice by longterm effects of dehydration

We observed a significant increase in blood urea nitrogen $(\mathrm{BUN}) / \mathrm{sCr}$ in RES $1 \mathrm{~W}(p<0.05)$, and there was an increasing tendency in RES 4W $(p=0.08)$ in the age-matched comparison analysis after prolonged dehydration (Fig. 5a).
To further investigate these results, we conducted comparison analysis of differentially expressed genes in the kidneys of RES 4W versus CON 4W. There were significant changes in basement membrane integrity markers. Renal epithelial basement membrane component (Col4a4; 1.6fold decrease), integrins (Itgb5 and Itgb6; 1.7-fold decreases), and molecules of cell adhesion including cell tight 


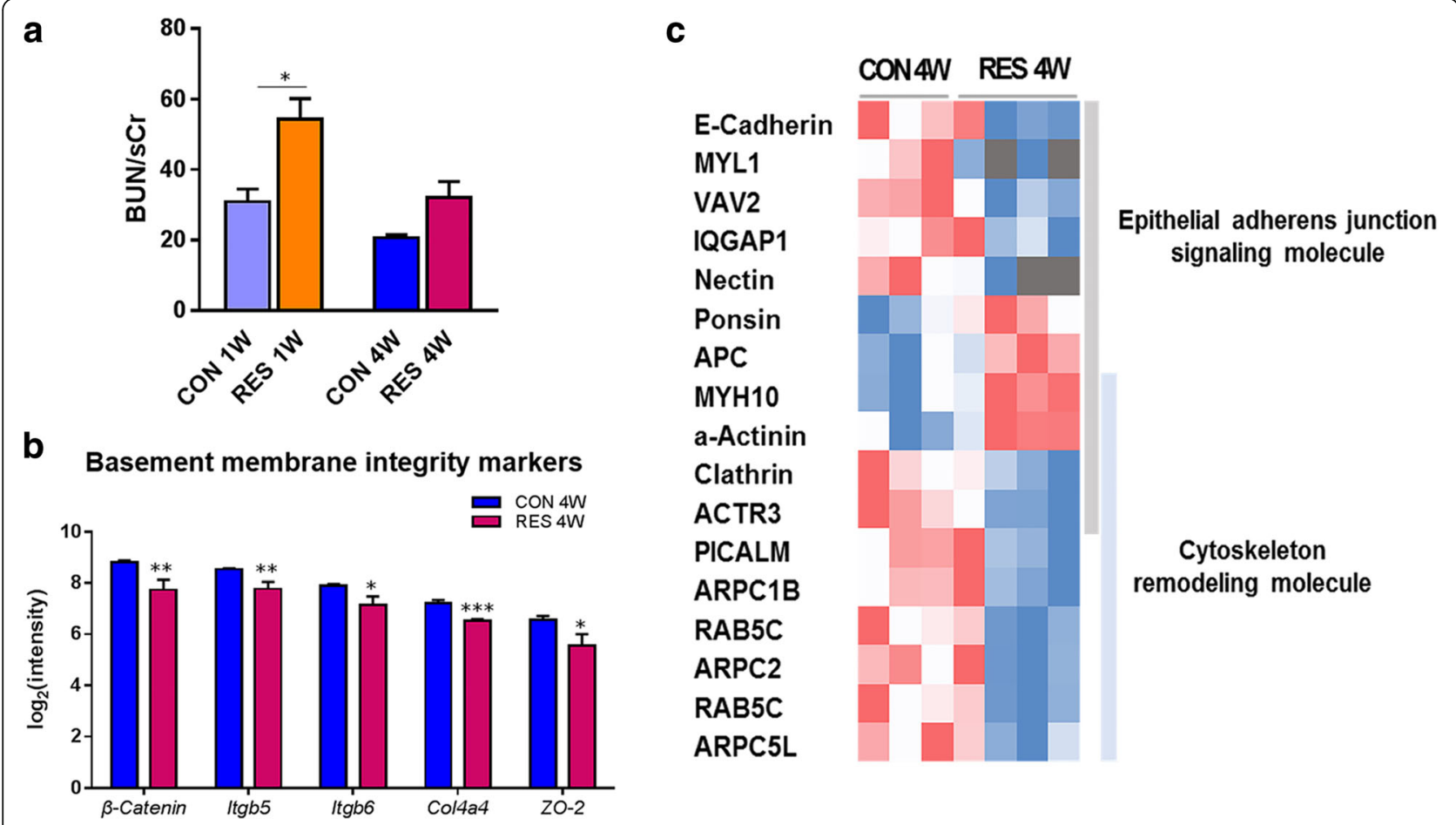

Fig. 5 Disorders in basement membrane integrity by prolonged dehydration. a An increase in BUN/sCr level in the dehydration group compared to the control group. b Dysregulation of basement membrane components at the transcript level. c The heatmap shows the signaling molecules related to cell-cell junction and cytoskeleton rearrangement underlying altered membrane integrity. Data are expressed as mean \pm SEM. Student's $t$ test; ${ }^{*} p<0.05,{ }^{* *} p<0.01,{ }^{* * *} p<0.001$ versus control group

junctions or adherens junctions ( $\beta$-catenin and $\mathrm{ZO}-2$; twofold decreases) were reduced at the transcription level (Fig. 5b). These results may suggest that prolonged dehydration ultimately gave rise to breakdown of basement membrane assembly in the kidneys of juvenile mice, which can be secondary cause to functional disorders and renal pathophysiology.

In order to understand the implicit mechanism underlying the deterioration of membrane integrity, we confirmed the top significant pathways by canonical pathway analysis. We found that transcriptional events related to renal structural integrity were intrinsically altered via dysregulation of the epithelial adherens junction and its remodeling pathway (Additional file 3 ). Cadherin (Cdh1)catenin (Ctnnb1) complex and nectin (Pvrl1) complex, which have roles in epithelial cell adhesion integrity and tissue homeostasis, were downregulated by sustained dehydration. Downstream molecules such as Iqgap1, Actr3, and Vav2 were also decreased at the transcription level, indicating that alteration was observed in the network between cell-cell and cell-extracellular matrix contacts (Fig. 5c, Additional file 3). Iqgap1 is a signaling molecule detected during the establishment of foot processes [27]. Especially, 3.4-fold increased expression of $\alpha$-actinin-4, which is accompanied by foot progress effacement in damaged podocytes [32], was observed in the RES 4W group. In concert with depressed junction structural stability, many of the differentially regulated genes in the dehydration group were related to adherens junction recycling. The downstream effect of junction structure remodeling is implicated in clathrin-mediated endocytosis, which appeared to be suppressed in the dehydration group (Fig. 5c). Clathrin-mediated endocytosis is implicated in the cycling of cellular adhesion proteins [35]. Clathrin $(C l t c)$, a mediator of vesicle formation during endocytosis of the cadherin complex, was decreased by 1.5 -fold at the expression level in RES 4W. A gene encoding clathrin recruiting protein, Picalm, also showed decreased expression levels (1.7-fold change). Rab5c, with roles in vesicle docking and trafficking, had reduced transcription levels (1.6-fold change) along with depressed fusion of endocytic vesicles (Fig. 5c). From these results, we demonstrate that the dynamics of cytoskeleton and adherens junction signaling, which account for the membrane integrity, were dysregulated at the transcriptional level as a consequence of prolonged dehydration.

\section{Discussion}

It was widely accepted that nephrogenesis completes during the embryonic period. However, recent studies unearthed 
new facts showing that later genetic events occur in postnatal renal growth. One study revealed that gene expression from E19.5 to P35 had a postnatal developmental pattern, showing a declining trend in cell differentiation and tissue development with aging in the mouse kidney [50]. In comparing the kidneys of adults to newborns, molecular transporters were found to be dominant in the transcriptome profiles [42]. Concurring with previous results, we identified that kidneys during infancy showed sequential processes indicating structural and functional maturation. Infantile kidneys, at 4 weeks old, were still differentiating and developed into functionally mature kidneys at 7 weeks old. This result provides critical evidence that postnatal care such as environmental and nutritional interventions is required for normal organ development since early renal dysfunction is implicated in various renal disorders in later life [13]. There is, however, a need for further studies to fully elucidate postnatal renal maturation using genetic perturbation animal models to elicit the importance of neonatal intervention for optimal growth.

Gene regulatory networks function in a coordinated fashion in kidney organogenesis to maintain kidney integrity [6]. It is, thus, emphasized that genetic developmental processes during the early postnatal life might be in charge of governing kidney health in later life. Moreover, despite the importance of adequate hydration in the maintenance of a healthier life, few studies have identified molecular mechanisms underlying the impacts of dehydration [17]. More than that, a fundamental methodological issue related to the experimental model of dehydration did not allow us to examine the true effects of dehydration. For instance, dehydration induced by acute water deprivation or heat and exercise stimulus could accompany or promote adverse physiological changes related to the dehydrating condition. Those also are incapable of generating consistent and controlled state of dehydration [28]. Here, we aimed to generate mild dehydration in animals, reproducing the condition of individuals that drink less water daily during the life cycle. With this approach, we were able to verify the molecular mechanisms underlying sustained moderate dehydration by profiling renal transcriptomes and anticipated its utility for the understanding of fundamental mechanisms underlying pediatric dehydration in the context of renal development.

We looked into the implications of dehydration throughout the early postnatal stage, from 4 to 7 weeks. Among corresponding mediators in kidney morphogenesis, the Shh signaling pathway and downstream target genes involved in the cell cycle and cell differentiation were downregulated as a consequence of dehydration. Shh is described as an important signaling molecule in renal patterning and the cell cycle during embryonic renal development [14]. Of its effectors, Glis1 has a possible regulatory role in retaining normal morphological integrity in the kidney [20,47]. Since the transcription level of Glis1 was reduced and its target genes were changed in their expressions in the dehydration group, it is possible that genetic events associated with cell proliferation, differentiation, and death were implicated under improper hydration conditions.

As dehydration progressed throughout the developmental period, we found the transcriptional network involved in tissue integrity was deteriorated. Kidney integrity in structure and function is characterized by a series of successive modifications from neonates to aged mice $[8,37]$. Sequential developmental processes in the kidneys correlate with numerous renal defects such as renal agenesis, hypoplasia, and polycystic kidney disease (PKD) [38]. In other words, normal renal organogenesis is necessary for mature functioning $[8,44,48,50]$ and diverse renal diseases are common consequences of abnormal kidney development $[8,38]$. That is, it is conceivable that consecutive dehydration along with perturbation of morphogenesis influences sequential developmental steps including system functional maturation. One possible mechanism responsible for functional injury in the hyperosmolar state is demonstrated by the observation that continuous angiotensin II infusion into rats induced injuries in various cell types with worsened renal function [18]. In line with this report, kidney-specific cellular organization that is composed of networks between podocytes and the glomerular basement membrane might be damaged as the renin-angiotensin system was activated (data not shown) in the dehydration group. Genes associated with glomerular and tubular architecture, which includes basement membrane, slit diaphragm, and junction structure showed different mRNA expression patterns. In addition, many genes related to adherens junction-cytoskeleton dynamics were shown to be downregulated by dehydration. In fact, precise regulation of junctional adhesion molecules is a decisive element in the assembly of the actin cytoskeleton, ensuring tissue homeostasis and recovery from injuries [11]. These adhesion molecules have pivotal roles in renal epithelial cell dedifferentiation, proliferation, and migration. It is likely that the disruption in structural maturation during infancy resulted in defects in morphological integrity in terms of the loss of cell-cell and cell-membrane contacts in juvenile kidneys and failed adaptation to the loss of cells during regeneration [4]. Meanwhile, once renal cells are injured, loss of intact cells is directly linked to the loss of nephrons [32]. Due to degenerative changes in cellular integrity along with podocyte foot effacement, a limited number of intact nephrons are exposed to hyperfiltration. Therefore, overload of glomerular flow per unit of the kidney might result in glomeruli injuries and subsequent renal 
functional perturbation, leading to increased $\mathrm{BUN} / \mathrm{sCr}$ ratio [10]. It is, therefore, conceivable that reduced membrane integrity impairs glomerular barrier function followed by improper tissue morphogenesis and homeostasis. However, it still needs histological and functional analysis to show direct evidence of physiological derangement or pathological changes of renal morphology in future studies.

Interestingly, prolonged dehydration during infancy resulted in reduced plasma creatinine levels. Based on present knowledge, reduction in the pool size or rate of creatinine metabolism, known as a "creatinine deficit," may account for the reduction in plasma creatinine under pathophysiologic conditions [12, 19]. Otherwise, we assume that failure in toxic substance clearance by renal insufficiency resulted in hyper toxic loads, augmenting systemic disorders in animals. While plasma creatinine is one of the most frequently used markers for kidney disease, it could blunt the early diagnosis of pediatric renal diseases in cases where the clinical marker is arbitrarily estimated. We, hereby, suggest that plasma creatinine might underestimate the significance of renal disorders in pediatric dehydration. Thus, it is noted that different clinical markers are required for pediatric renal disorders. Meanwhile, at the onset of dehydration, dehydrated mice exhibited reduced diet consumption during the first few days (Additional file 4). Despite catching up to normal diet intake after the first week of the dehydration experiment, insufficient energy intake could be a cause of developmental disorders in the situation of impaired renal development in pediatric dehydration. Notwithstanding, it is noted that animals have different patterns of eating behavior with reduced appetite under high osmolar stimulus while they are subjected to strict control of osmolality [15]. Given the complexity of the physiological response, therefore, we suggest that changes in eating behavior are a validated consequence of physiological adaptation to water deprivation [15].

In this research, we demonstrate transcriptional events during renal development in infancy and show that the impacts of inadequate water intake in the early postnatal stage heavily rely on the impairment of normal renal maturation. This finding suggests that optimal nutritional intervention would be required for successful renal development.

\section{Conclusions}

Altogether, this study gives us better insights into the effects of dehydration on functionally immature kidneys of infants and may provide possible markers for clinical applications in pediatric dehydration. In this respect, this study could be a cornerstone providing predictive biomarkers for future examination of known renal diseases and a broadened perspective of pediatric renal defects during development.

\section{Additional files}

\begin{abstract}
Additional file 1: Differentially expressed genes during normal renal development from infancy to juvenile period. Differentially expressed genes in a comparison analysis between CON 1W and CON 4W group, with statistical significant change $(p<0.05$ and fold change $>1.5)$. (XLSX 24 kb)
\end{abstract}

Additional file 2: Upstream regulators in infantile renal development. Upstream regulator analysis shows the top predicted regulators that connected to the downstream nodes. Activation z-score of each regulator is presented in brackets. Edges indicate predicted relationships, colored with yellow and blue when it led to activation and inhibition, respectively. (TIF 8929 kb)

Additional file 3: Dysregulation of transcriptional network associated with cell junction dynamics. (A) Top canonical pathways that were differentially regulated following dehydration in juvenile mice. (B) Suppression of adherens junction signaling accounts for altered cytoskeleton rearrangement, which caused alteration of glomerular barrier integrity. Genes in green and red color were down- and upregulated, respectively. (TIF $5873 \mathrm{~kb}$ )

Additional file 4: Diet intake during experimental period. Animals in the dehydration group exhibited reduced diet consumption during the first few days but caught up to normal diet intake after the first week of the dehydration experiment. Data are expressed as mean \pm SEM. Student's $t$ test; ${ }^{*} p<0.05$ versus control group. (TIF $45 \mathrm{~kb}$ )

\section{Abbreviations \\ Avp: Arginine vasopressin; BUN: Blood urea nitrogen; ECM: Extracellular matrix GO: Gene ontology; GSEA: Gene set enrichment analysis; PCA: Principal component analysis; PKD: Polycystic kidney disease; sCr: Serum creatinine; $\mathrm{SHH}$ : Sonic hedgehog}

\section{Acknowledgements}

This work was supported by the Coway Co., Ltd.

\section{Availability of data and materials}

Microarray dataset is available at the Gene Expression Omnibus (www.ncbi.nlm.nih.gov/geo/, accession number GSE75604).

\section{Authors' contributions}

DS designed and conceived the study and helped to draft the manuscript. CK carried out the experiments (animal care, biochemical and gene expression analysis, and Western blotting) and the statistical analysis and drafted the manuscript. Both authors read and approved the final manuscript.

\section{Competing interests}

The authors declare that they have no competing interests.

Ethics approval and consent to participate

All experimental procedures were approved by the IACUC (Institutional Animal Care and Use Committee) of Seoul National University and conducted according to the IACUC guidelines.

Received: 10 March 2016 Accepted: 3 October 2016

Published online: 20 October 2016

References

1. Bates CM, Kharzai S, Erwin T, Rossant J, Parada LF. Role of N-myc in the developing mouse kidney. Dev Biol. 2000;222:317-25.

2. Baxter J, Yoffey J. The post-natal development of renal tubules in the rat. J Anat. 1948:82:189.

3. Berschneider B, Königshoff M. WNT1 inducible signaling pathway protein 1 (WISP1): a novel mediator linking development and disease. Int J Biochem Cell Biol. 2011;43:306-9. 
4. Bonventre JV. Dedifferentiation and proliferation of surviving epithelial cells in acute renal failure. J Am Soc Nephrol. 2003;14:S55-61.

5. Boutet A, De Frutos CA, Maxwell PH, Mayol MJ, Romero J, Nieto MA. Snail activation disrupts tissue homeostasis and induces fibrosis in the adult kidney. EMBO J. 2006;25:5603-13.

6. Burrow CR. Regulatory molecules in kidney development. Pediatr Nephrol. 2000;14:240-53.

7. Clark SL. Cellular differentiation in the kidneys of newborn mice studied with the electron microscope. J Biophys Biochem Cytol. 1957;3:349-62.

8. Čukuranović R, Vlajković S. Age related anatomical and functional characteristics of human kidney. Organ. 2005;7:14.

9. D'Anci KE, Constant F, Rosenberg $\mathrm{H}$. Hydration and cognitive function in children. Nutr Rev. 2006;64:457-64.

10. de Barros Sene L, Mesquita FF, de Moraes LN, Santos DC, Carvalho R, Gontijo JAR, Boer PA. Involvement of renal corpuscle microRNA expression on epithelial-to-mesenchymal transition in maternal low protein diet in adult programmed rats. PLoS One. 2013;8(8):e71310.

11. Denker BM, Sabath E. The biology of epithelial cell tight junctions in the kidney. J Am Soc Nephrol. 2011;22:622-5.

12. Doi K, Yuen PS, Eisner C, Hu X, Leelahavanichkul A, Schnermann J, Star RA. Reduced production of creatinine limits its use as marker of kidney injury in sepsis. J Am Soc Nephrol. 2009;20:1217-21.

13. Dressler GR. Advances in early kidney specification, development and patterning. Development. 2009;136:3863-74.

14. Gill PS, Rosenblum ND. Control of murine kidney development by sonic hedgehog and its GLI effectors. Cell Cycle. 2006;5:1426-30.

15. Greenwood MP, Mecawi AS, Hoe SZ, Mustafa MR, Johnson KR, Al-Mahmoud GA, Elias LL, Paton JF, Antunes-Rodrigues J, Gainer H. A comparison of physiological and transcriptome responses to water deprivation and salt loading in the rat supraoptic nucleus. Am J Phys Regul Integr Comp Phys. 2015;308:R559-68.

16. Humphreys BD. Kidney structures differentiated from stem cells. Nat Cell Biol. 2014;16:19-21.

17. Jéquier $E$, Constant F. Water as an essential nutrient: the physiological basis of hydration. Eur J Clin Nutr. 2010;64:115-23.

18. Johnson RJ, Alpers CE, Yoshimura A, Lombardi D, Pritzl P, Floege J, Schwartz SM. Renal injury from angiotensin II-mediated hypertension. Hypertension. 1992;19:464-74.

19. Jones JD, Burnett PC. Creatinine metabolism in humans with decreased renal function: creatinine deficit. Clin Chem. 1974;20:1204-12.

20. Kang HS, ZeRuth G, Lichti-Kaiser K, Vasanth S, Yin Z, Kim Y-S, Jetten AM. Glisimilar (Glis) Krüppel-like zinc finger proteins: insights into their physiological functions and critical roles in neonatal diabetes and cystic renal disease. Histol Histopathol. 2010;25:1481

21. Kim T-M, Ramírez V, Barrera-Chimal J, Bobadilla NA, Park PJ, Vaidya VS. Gene expression analysis reveals the cell cycle and kinetochore genes participating in ischemia reperfusion injury and early development in kidney. PLoS One. 2011;6:e25679.

22. Krämer A, Green J, Pollard J, Tugendreich S. Causal analysis approaches in ingenuity pathway analysis (IPA). Bioinformatics. 2013;29:btt703.

23. Kuure S, Vuolteenaho R, Vainio S. Kidney morphogenesis: cellular and molecular regulation. Mech Dev. 2000;92:31-45.

24. Larsson L, Aperia A, Wilton P. Effect of normal development on compensatory renal growth. Kidney Int. 1980;18:29-35.

25. LeBleu VS, MacDonald B, Kalluri R. Structure and function of basement membranes. Exp Biol Med. 2007;232:1121-9.

26. Lee DB, Huang E, Ward HJ. Tight junction biology and kidney dysfunction. Am J Physiol Renal Physiol. 2006;290:F20-34

27. Lehtonen S, Ryan JJ, Kudlicka K, lino N, Zhou H, Farquhar MG. Cell junctionassociated proteins IQGAP1, MAGI-2, CASK, spectrins, and a-actinin are components of the nephrin multiprotein complex. Proc Natl Acad Sci U S A. 2005;102:9814-9.

28. Lieberman HR. Hydration and cognition: a critical review and recommendations for future research. J Am Coll Nutr. 2007;26:555S-61.

29. Masento NA, Golightly M, Field DT, Butler LT, van Reekum CM. Effects of hydration status on cognitive performance and mood. Br J Nutr. 2014; 111:1841-52

30. McKinley MJ, Johnson AK. The physiological regulation of thirst and fluid intake. Physiology. 2004;19:1-6.
31. Muller U, Brandli A. Cell adhesion molecules and extracellular-matrix constituents in kidney development and disease. J Cell Sci. 1999; 112:3855-67.

32. Pavenstädt H, Kriz W, Kretzler M. Cell biology of the glomerular podocyte. Physiol Rev. 2003;83:253-307.

33. Popkin BM, D'Anci KE, Rosenberg $H$. Water, hydration, and health. Nutr Rev. 2010;68:439-58

34. Quinn L, Latham S, Kalionis B. The homeobox genes MSX2 and MOX2 are candidates for regulating epithelial-mesenchymal cell interactions in the human placenta. Placenta. 2000;21:S50-4.

35. Rappoport JZ, Simon SM. Real-time analysis of clathrin-mediated endocytosis during cell migration. J Cell Sci. 2003;116:847-55.

36. Reiser J, Kriz W, Kretzler M, Mundel P. The glomerular slit diaphragm is a modified adherens junction. J Am Soc Nephrol. 2000;11:1-8.

37. Rodriguez-Soriano J, Vallo A, Castillo G, Oliveros R. Renal handling of water and sodium in infancy and childhood: a study using clearance methods during hypotonic saline diuresis. Kidney Int. 1981;20:700-4.

38. Schedl A. Renal abnormalities and their developmental origin. Nat Rev Genet. 2007:8:791-802

39. Schwab K, Patterson LT, Aronow BJ, Luckas R, Liang H-C, Potter SS. A catalogue of gene expression in the developing kidney. Kidney Int. 2003:64:1588-604

40. Shannon P, Markiel A, Ozier O, Baliga NS, Wang JT, Ramage D, Amin N, Schwikowski B, Ideker T. Cytoscape: a software environment for integrated models of biomolecular interaction networks. Genome Res. 2003;13:2498-504.

41. Shon W-J, Lee Y-K, Shin JH, Choi EY, Shin D-M. Severity of DSS-induced colitis is reduced in Ido1-deficient mice with down-regulation of TLRMyD88-NF-kB transcriptional networks. Sci Rep. 2015;5:17305.

42. Stuart RO, Bush KT, Nigam SK. Changes in global gene expression patterns during development and maturation of the rat kidney. Proc Natl Acad Sci. 2001;98:5649-54.

43. Sturn A, Quackenbush J, Trajanoski Z. Genesis: cluster analysis of microarray data. Bioinformatics. 2002;18:207-8.

44. Thiagarajan RD, Georgas KM, Rumballe BA, Lesieur E, Chiu HS, Taylor D, Tang D, Grimmond SM, Little MH. Identification of anchor genes during kidney development defines ontological relationships, molecular subcompartments and regulatory pathways. PLoS One. 2011;6:e17286.

45. Uhlenhaut $\mathrm{NH}$, Treier M. Transcriptional regulators in kidney disease: gatekeepers of renal homeostasis. Trends Genet. 2008;24:361-71.

46. Vainio S, Lin Y. Coordinating early kidney development: lessons from gene targeting. Nat Rev Genet. 2002;3:533-43.

47. Vasyutina E, Treier M. Molecular mechanisms in renal degenerative disease Seminars in Cell \& Developmental Biology. Acad Press. 2010;21:831-7.

48. Welham SJ, Wade A, Woolf AS. Protein restriction in pregnancy is associated with increased apoptosis of mesenchymal cells at the start of rat metanephrogenesis. Kidney Int. 2002;61:1231-42.

49. Welsh Gl, Saleem MA. The podocyte cytoskeleton-key to a functioning glomerulus in health and disease. Nat Rev Nephrol. 2012;8:14-21.

50. Wu B, Sahoo D, Brooks JD. Comprehensive gene expression changes associated with mouse postnatal kidney development. J Urol. 2013;189: 2385-90.

\section{Submit your next manuscript to BioMed Central and we will help you at every step:}

- We accept pre-submission inquiries

- Our selector tool helps you to find the most relevant journal

- We provide round the clock customer support

- Convenient online submission

- Thorough peer review

- Inclusion in PubMed and all major indexing services

- Maximum visibility for your research

Submit your manuscript at www.biomedcentral.com/submit 